\title{
Qualidade de vida, nível de atividade física e mobilidade funcional entre idosos institucionalizados e domiciliados
}

\author{
Quality of Life, Physical Activity Level and Functional Mobility among \\ Institutionalized and Homebound Elderly People
}

\author{
JÉSSICA LUANA DORNELLES DA COSTA ${ }^{1}$ \\ CARLOS LEANDRO TIGGEMANN ${ }^{2}$ \\ CAROLINE PIETADIAS ${ }^{3}$
}

\section{RESUMO}

Objetivo: comparar a qualidade de vida, os níveis de atividade física e a mobilidade funcional entre idosos institucionalizados e idosos domiciliados. Materiais e Métodos: trata-se de uma pesquisa quantitativa, descritiva e ex-post facto, realizada com 32 idosos, divididos em dois grupos: residentes em instituições $(n=16)$ e residentes em domicílio $(n=16)$. A seleção do grupo institucionalizado se deu por meio de sorteio de três das seis instituições existentes de um município do Vale do Taquari-RS, enquanto que os idosos domiciliados foram recrutados por meio de divulgação nos mesmos bairros em que estavam localizadas as instituições escolhidas. Para mensurar a qualidade de vida utilizou-se o Questionário Genérico SF-36, para o nível de atividade física o Questionário Internacional de Atividade Física e para a mobilidade funcional o Teste Timed Up-and-Go. Os testes estatísticos MannWhitney, Qui quadrado e Teste $T$ independente foram utilizados para análise estatística dos dados. Resultados: tanto a qualidade de vida em seus oito domínios como a mobilidade funcional, foram similares entre os grupos $(p>0,05)$. Quanto ao nível de atividade física, $100 \%$ dos idosos institucionalizados e $37,5 \%$ dos domiciliados foram considerados sedentários, sendo esta diferença significativa $(p<0,05)$. Conclusão: observou-se que o fato do idoso residir em sua residência ou em instituições específicas, não interfere na sua qualidade de vida e na sua mobilidade funcional. Já quanto ao nível de atividade física, ficou constatado que idosos domiciliados são mais ativos quando comparado aos seus pares.

\section{DESCRITORES}

Idoso. Qualidade de vida. Atividade motora.

\begin{abstract}
Objective: To compare the quality of life, physical activity level and functional mobility among institutionalized and homebound elderly people. Material and Methods: This was a quantitative, descriptive and ex-post facto study developed with 32 elderly people divided into two groups: resident in institutions $(n=16)$ and resident at home $(n=16)$. The selection of the institutionalized group was made by drawing lots from three of the six institutions of Vale do Taquari-RS, while the homebound group was recruited through the same districts in which the chosen institutions were located. The SF-36 Generic Questionnaire was used to measure quality of life, while the International Physical Activity Questionnaire was used to measure physical activity level, and the Timed Upand-Go Test was employed for functional mobility. MannWhitney, Chi-square and independent T-tests were used for statistical analysis. Results: Both quality of life, in its eight domains, and functional mobility, were similar between groups ( $p>0.05$ ). Regarding the level of physical activity, $100 \%$ of the institutionalized and $37.5 \%$ of the homebound elderlies were considered sedentary, with a significant difference $(p$ $<0.05)$. Conclusion: Living in their residence or in specific institutions does not interfere with the elderlies' quality of life and functional mobility. Nevertheless, with regard to physical activity, homebound elderlies were found to be more active as compared to their peers.
\end{abstract}

\section{DESCRIPTORS}

Aged. Quality of life. Motor Activity.

Graduanda em Educação Física Bacharelado, Centro Universitário Univates, Lajeado, RS, Brasil.

2 Coordenador do Laboratório de Fisiologia do Exercício do Centro Universitário Univates Lajeado, RS, Brasil. Faculdade da Serra Gaúcha (FSG) Grupo de Pesquisa e Estudos em Saúde e Performance, Caxias do Sul, RS, Brasil.

3 Pesquisadora do Laboratório de Pesquisa do Exercício da Universidade Federal do Rio Grande do Sul (UFRGS), Porto Alegre, RS, Brasil. Faculdade da Serra Gaúcha (FSG) - Grupo de Pesquisa e Estudos em Saúde e Performance, Caxias do Sul, RS, Brasil. 
envelhecimento populacional foi um fenômeno inicialmente observado em países desenvolvidos, mas atualmente, é nos países em desenvolvimento que a população idosa tem aumentado de forma acelerada ${ }^{1}$. O aumento da população idosa no Brasil é um exemplo dessa afirmativa, estimando-se 32 milhões de pessoas idosas para $2025^{2}$. O envelhecer é um processo biológico, no qual ocorrem alterações na estrutura física do indivíduo, fisiológicas com diminuição das capacidades funcionais, e também por mudanças nos aspectos psicológicos e sociais ${ }^{3}$. Associado a isso, o aumento do percentual de pessoas sedentárias a partir dos 65 anos de idade pode agravar ainda mais estes efeitos ${ }^{4}$.

Vários estudos vêm mostrando a importância da atividade física na vida desta população, sendo que os benefícios são muitos, sobretudo na melhoria da qualidade de vida em diferentes domínios, sejam eles biológicos, sociais ou psicológicos ${ }^{5,6}$. No entanto, dentre as mais diversas preocupações relacionadas ao desafio de proporcionar uma vida saudável e com qualidade, a moradia também é um aspecto importante na vida de um idoso, sendo representada pelo domicílio em que está inserido, ou ainda, em instituições de longa permanência.

Estas instituições podem assumir diversas funções, como a responsabilidade de dar proteção e alimentação aos idosos que foram rejeitados pelas suas famílias e sociedade. Possuindo uma organização formal e com regras estabelecidas, tendo em comum a questão de oferecer atendimento à saúde e algumas atividades de ocupação ou lazer $^{7}$. Em contraponto a esta necessidade, um estudo ${ }^{8}$ constatou que após apenas três meses de permanência de idosos em uma instituição de longa permanência, os mesmos tiveram uma diminuição significativa de sua funcionalidade.

Outra opção de moradia para o idoso é o domicílio, o qual é o lugar em que muitos vivem com suas famílias, sozinhos ou com algum outro acompanhante. Este ambiente foi construído ao longo de toda vida, contendo características pessoais, sociais, culturais, normas do próprio domicílio, questões relacionadas à funcionalidade e diversos outros fatores, o que torna este ambiente caracterizado de acordo com as pessoas que convivem no mesmo 9 .

Assim, parece evidente que as distintas maneiras de residência podem influenciar na saúde dos idosos. Idosos institucionalizados têm capacidade funcional e qualidade de vida menor quando comparados com os não institucionalizados ${ }^{10}$. Assim, considerando o reduzido número de pesquisas com esta temática, este estudo tem como objetivo comparar a qualidade de vida, os níveis de atividade física e a mobilidade funcional entre idosos institucionalizados e idosos domiciliados.

\section{METODOLOGIA}

Este estudo caracteriza-se como pesquisa quantitativa, descritiva e ex-post facto, tendo seguido a Resolução 466/12 com pesquisa em seres humanos e aprovado pelo Comitê em Ética e Pesquisa do Centro Universitário Univates (parecer 673.477). Para a coleta dos dados, foi apresentado aos responsáveis das instituições o Termo de Anuência, e aos participantes o Termo de Consentimento Livre Esclarecido, onde foram informados sobre os objetivos e procedimentos desta investigação.

A amostra foi constituída de forma não probabilística por acessibilidade, sendo constituída por 32 idosos com idade média de 76,82 18,01 anos, divididos em dois grupos, sendo 16 institucionalizados e 16 domiciliados, ambos apresentando $75 \%$ de mulheres, residentes em um município do Vale do Taquari - RS. Os critérios para inclusão do estudo foram: (1) idade igual ou superior a 60 anos, (2) que apresentassem capacidade de se locomoverem de forma independente, (3) não participante de grupos regulares de exercícios físicos e (4) com capacidade cognitiva preservada. Como critério de exclusão foi determinado a não realização completa das avaliações, sendo que nenhum sujeito foi excluído. A seleção das instituições para o estudo se deu por meio de sorteio, sendo sorteadas três das seis instituições existentes no município. Após a definição da quantidade de idosos das instituições $(n=16)$, foi definido o mesmo número de participantes para os idosos em domicílios. O recrutamento dos idosos domiciliados ocorreu por meio de divulgação (redes sociais, panfletos, ligações, outros) nos mesmos bairros em que estavam localizadas as instituições escolhidas. Em seguida ocorreu a visita às residências dos mesmos, sendo apresentados os objetivos do estudo, e verificado a obediência aos critérios de inclusão. Uma vez confirmado a possibilidade de participação, os sujeitos assinavam o termo de consentimento livre esclarecido e iniciavam-se as coletas.

Para a avaliação do nível de atividade física, 
utilizou-se o Questionário Internacional de Atividade Física (IPAQ) ${ }^{11}$. A versão utilizada foi a forma curta, encontrando-se três questões relacionadas ao tempo que as pessoas se dedicaram fazendo atividade física na última semana. As mesmas são classificadas como caminhada, atividades moderadas que necessitem de algum esforço físico, e atividades vigorosas, nas quais seja necessário um grande esforço físico. Para o tratamento das respostas, aplicaram-se os critérios específicos do instrumento, tendo a classificação final do participante em fisicamente inativo (sedentário), fisicamente ativo de forma moderada ou intensa.

Para a avaliação da mobilidade funcional foi usado o Teste Timed Up-and-Go (TUG), criado com o propósito de avaliar o equilíbrio sentado, de sentado colocar-se na posição de pé e no caminhar $^{12}$. O teste é iniciado com o participante sentado em uma cadeira (altura da cadeira $46 \mathrm{~cm}$ ), mantendo as costas apoiadas no encosto; após o comando "já", o mesmo coloca-se na posição de pé, caminhar por três metros, contornar um objeto (cone), retornar e sentar-se novamente, sendo este trajeto cronometrado. Conforme os critérios sugeridos para este teste, os participantes que realizaram este teste em até 10 segundos foram considerados sem alteração no equilíbrio e com baixo risco de quedas; entre 11 e 20 segundos, são idosos sem alteração importante no equilíbrio, mas apresentam alguma fragilidade e médio risco de quedas; mais de 20 e menos de 30 segundos são idosos que necessitam de intervenção; acima de 30 segundos, idosos com alto risco de quedas e indivíduos dependentes em atividades de vida diária $^{13}$

Para verificar indicadores de qualidade de vida, utilizou-se o questionário genérico SF - 36
(Medical Outcomes Study 36 - Item Short - Form Health Survey) ${ }^{14}$. O mesmo é formado por 36 itens, dividido em oito domínios: capacidade funcional, aspectos físicos, dor, estado geral de saúde, vitalidade, aspectos sociais, aspectos emocionais e saúde mental. Para o tratamento das respostas foi utilizado os critérios específicos do instrumento, apresentando um escore de 0 a 100, sendo zero o pior estado geral de saúde e 100 o melhor.

A análise estatística dos dados constituiu da análise descritiva com média, desvio padrão e distribuição de frequência. Para a comparação dos domínios de qualidade de vida, utilizou-se o teste U-Mann-Whitney e para os percentuais de classificação dos níveis de atividade física o teste Qui quadrado. Os valores do teste TUG foram comparados por meio do teste T independente, uma vez assumida a normalidade (Teste de Shapiro-Wilk) e homogeneidade (Teste de Levene) dos valores. Todos os procedimentos foram realizados no software SPSS v. 18.0, sendo adotado um nível de significância de á<0,05.

\section{RESULTADOS}

Quanto ao nível de atividade física, o grupo institucionalizado apresentou um percentual superior de sedentários, quando comparado aos idosos domiciliados $(100 \%$ vs $37,5 \%$, respectivamente; $p<0,05)$. Não foram encontradas diferenças quanto à mobilidade funcional entre idosos institucionalizados e domiciliados $(15,99 \pm 4,34$ s vs $13,69 \pm 4,27 s$, respectivamente; $p>0,05)$. Em relação aos resultados obtidos sobre a qualidade de vida dos idosos, pôde-se constatar que os grupos apresentaram valores similares $(p>0,05)$ nos oito domínios da qualidade de vida (Tabela 1).

\begin{tabular}{lll}
\hline $\begin{array}{l}\text { Tabela 1: Comparação entre os idosos institucionalizados e domiciliados nos domínios da } \\
\text { qualidade de vida (média } \pm \text { desvio padrão). }\end{array}$ & $\begin{array}{l}\text { Institucionalizados } \\
(\mathbf{n = 1 6 )}\end{array}$ & $\begin{array}{l}\text { Domiciliados } \\
\mathbf{( n = 1 6 )}\end{array}$ \\
& $40,63 \pm 26,64$ & $54,69 \pm 23,77$ \\
Capacidade funcional & $46,88 \pm 41,71$ & $70,32 \pm 30,58$ \\
Limitações por aspectos físicos & $67,57 \pm 26,59$ & $61,07 \pm 26,84$ \\
Dor & $55,50 \pm 21,41$ & $51,44 \pm 16,71$ \\
Estado geral de saúde & $62,50 \pm 21,99$ & $64,07 \pm 13,81$ \\
Vitalidade & $93,75 \pm 15,14$ & $82,82 \pm 22,77$ \\
Aspectos sociais & $93,75 \pm 25,00$ & $83,33 \pm 29,82$ \\
Limitação por aspectos emocionais & $77,25 \pm 20.44$ & $66,25 \pm 17,89$ \\
Saúde mental & & \\
\hline
\end{tabular}




\section{DISCUSSÃO}

Os principais resultados deste estudo indicam que os idosos institucionalizados são mais sedentários que seus pares domiciliados, sendo que a qualidade de vida e a mobilidade funcional foram semelhantes entre ambos os grupos. Em relação aos baixos níveis de atividade física dos idosos nas instituições, estudo ${ }^{15}$ constatou que no município de Natal - RN, uma das instituições pesquisadas apresentou $100 \%$ dos idosos como sedentários.

A falta de promoção de atividades físicas possibilita um aumento da quantidade de idosos institucionalizados sedentários, podendo também ser evidenciado em pesquisa ${ }^{16}$, onde constatou que a maioria das instituições de amparo ao idoso que fizeram parte do estudo, não promoviam a prática de atividades físicas regulares aos idosos. Ainda, nenhuma das instituições avaliadas dispunha de profissionais de Educação Física em seu quadro pessoal, e além disso, não havia nenhuma programação durante a semana de atividades que envolvessem atividades físicas gerais como por exemplo, caminhadas, trabalhos de jardinagem, atividades domésticas, e outras.

Em relação aos idosos domiciliados, no presente estudo foi adotado como critério de inclusão que os mesmos não estivessem participando de programas regulares de exercícios físicos, e mesmo assim, mais de $60 \%$ dos mesmos foram considerados fisicamente ativos, tendo as tarefas domésticas como principal promotor do movimento corporal. A importância destas tarefas no dia a dia de idosos também está descrita em outro estudo ${ }^{17}$, onde embora os idosos institucionalizados participassem de um programa de atividade física, os domiciliados apresentaram níveis significativamente maiores de atividades físicas, pois realizam tarefas domésticas diárias em suas rotinas, enquanto os idosos institucionalizados não são contemplados com essas atividades.

No que se refere ao resultado da mobilidade funcional, percebe-se que ambos os grupos apresentaram resultados similares, e classificaramse sem alteração importante de equilíbrio, mas apresentando alguma fragilidade e médio risco de quedas. A literatura tem indicada que a simples permanência de idosos em instituições de longa permanência, já pode contribuir no decréscimo da funcionalidade dos mesmos ${ }^{8}$ Com a mobilidade diminuída, o indivíduo está mais vulnerável a risco de quedas e dependência ${ }^{18}$. Vários estudos ${ }^{19,20,21}$ têm confirmado que a prática regular de atividade física promove muitos benefícios, em especial com o treinamento de força. Em um destes estudos ${ }^{19}$, foi verificado que os idosos que realizaram treinamento de força por apenas 12 semanas, tiveram melhoras significativas no aspecto funcional com incrementos chegando a até $23 \%$ após este período.

Em relação à qualidade de vida, sabe-se que a mesma vem sendo muito estudada, em especial na população idosa. No presente estudo, todos os oito domínios apresentaram valores similares entre idosos institucionalizados e domiciliados. Estes resultados são semelhantes aos encontrados na literatura ${ }^{22}$, onde, mesmo os idosos institucionalizados apresentando média de idade superior aos domiciliados, a qualidade de vida foi semelhante entre os grupos. Em contrapartida, estudos ${ }^{23,24}$ constataram que em todos os seis domínios da qualidade de vida avaliados, o grupo institucionalizado apresentou grau de satisfação inferior ao grupo domiciliado, sendo este resultado justificado pelos autores possivelmente pela maior capacidade de tomada de decisões e participação social destes sujeitos.

Assim, apesar da semelhança encontrada entre os grupos nos diferentes domínios da qualidade de vida, pode-se perceber pequenas alterações entre os domínios nos respectivos grupos. De forma geral, observa-se que os domínios relativos às questões sociais, mentais e emocionais, apresentaram escores mais altos, sendo estes achados corroborados por outros estudos que também encontraram respostas semelhantes ${ }^{15-2-23}$. Assim, tanto nas instituições como em domicílios, parece existir boas relações sociais e poucas limitações emocionais entre os idosos.

Em relação à atividade física, os estudos têm demonstrado que idosos ativos apresentam melhores resultados nos domínios da qualidade de vida $^{5,25}$, em especial naqueles relacionados à capacidade funcional, limitações por aspectos físicos e vitalidade. Constatando ${ }^{26}$ que indivíduos considerados ativos e praticantes de exercícios físicos têm uma melhor qualidade de vida, quando comparados a idosos ativos, mas não praticantes 
de exercícios físicos. Assim, é importante observar que a simples manutenção das atividades habituais elevadas (caminhadas, trabalhos de jardinagem e andar de bicicleta), não garantem a manutenção de níveis satisfatórios de força muscular e aspectos funcionais, e consequentemente, na qualidade de vida $^{27,28}$. Portanto, um programa de exercícios físicos direcionados para pessoas idosas pode contribuir para um estilo de vida independente e mais saudável, tendo como objetivo a melhora da capacidade física, o aumento do contato social e a redução de problemas psicológicos, de ansiedade e depressão ${ }^{29}$.

A comparação do ambiente em que os idosos residem possui uma grande complexidade, podendo sofrer a influência de vários aspectos de difícil controle, como por exemplo, do perfil das pessoas com quem convive em seus domicílios ou os profissionais disponíveis nas instituições. Possivelmente, estudos longitudinais poderiam apresentar uma melhor respostas à estas questões, bem como, a investigação de uma maior quantidade de sujeitos.

\section{CONCLUSÃO}

Como conclusão deste estudo, pode-se constatar que o fato do idoso residir em sua residência ou em instituições específicas, não interfere na sua qualidade de vida e na sua mobilidade funcional. Já quanto ao nível de atividade física, ficou constatado que idosos domiciliados são mais ativos quando comparado aos seus pares institucionalizados.

\section{REFERENCIAS}

1. Costa MFFL, Guerra HL, Barreto SM, Guimarães RM. Diagnóstico da situação de saúde da população brasileira: um estudo da mortalidade e das internações hospitalares públicas. Inf. Epidemiol. Sus. 2000; 9(1):2341.

2. Brasil. Ministério da Saúde: envelhecimento e saúde da pessoa idosa [online]; 2007; Brasília. Cadernos de atenção básica. Brasília: 2007.

3. Okuma SS. O idoso e a atividade física: fundamentos e pesquisa. Campinas: Papirus; 1998.

4. Brasil. Vigilância de Fatores de Risco e Proteção para Doenças Crônicas por Inquérito Telefônico [online]; 2013.

5. Toscano JJO, Oliveira ACC. Qualidade de vida em idosos com distintos níveis de atividade física. Rev. Bras. Med. Esporte. 2009; 15(3):169-73.

6. Mazo GZ, Lopes MA, Benedetti TB. Atividade física e o idoso: concepção gerontologia. Porto Alegre: Sulina; 2009.

7. Cortelletti IA, Casara MB, Herédia VBM. Idoso asilado: um estudo gerontológico. Caxias do Sul: Educs/ Edipucrs; 2004

8. Oliveira ER, Silva RR, Carvalho AGC, Carvalho SMCC, Maciel LFM, Alves LFM, et al. Capacidade Funcional de Idosas de Instituição de Longa Permanência no Município de João Pessoa, Paraíba, Brasil: Estudo Piloto. Rev. bras. ciênc. saúde. 2012; 16(2):83-88.

9. Duarte YAO, Diogo ME. Atendimento domiciliar: um enfoque gerontologico. São Paulo: Atheneu; 2000.

10. Mincato PC, Freitas CLR. Qualidade de vida dos idosos residentes em instituições asilares da cidade de Caxias do Sul - RS. RBCEH. 2007; 4(1):127-38.

11. IPAQ. Guidelines for Data Processing and Analysis of the. International Physical Activity Questionnaire. 2005.
12. Rikli RE, Jones JC. Functional Fitness Normative Scores for Community-Residing Older Adults, Ages 60-94. Journal of Aging and Physical Activity. 1999; 7(2):162 81.

13. Almeida ST, Soldera CLC, Carli GA, Gomes I, Resende TL. Análise de fatores extrínsecos e intrínsecos que predispõem a quedas em idosos. Rev. Assoc. Méd. Bras. 2012; 58(4):427-33.

14. Ciconelli RM, Ferraz MB, Santos W, Meinão I, Quaresma MR. Tradução para a língua portuguesa e validação do questionário genérico de avaliação de qualidade de vida SF-36 (Brasil SF-36). Rev. Bras. de Reumatol. 1999; 39(3):143-50.

15. Davim RMB, Torres GV, Dantas SMM, Lima VM. Estudo com idosos de instituiç̃es asilares no município de Natal/RN: características socioeconômicas e de saúde. Rev. Latino-Am. Enfermagem. 2004; 12(3):51824.

16. Pereira SD, Guerra I. Análise do nível de atividade física nos asilos e instituições de apoio aos idosos na cidade de Juazeiro do Norte/CE: Conexões-Ciência e Tecnologia. 2010; 4(1):9-16.

17. Corazza DI. Influência da prática regula de atividade física sobre sintomas depressivos em idosos institucionalizados. [Dissertação]. Rio Claro: Universidade Estadual Paulista; 2005.

18. Ferrantin AC, Borges CF, Morelli JGS, Rebelatto JR. A execução de AVDS e mobilidade funcional em idosos institucionalizados e não-institucionalizados. Fisioterapia em Movimento. 2007; 20(3):115-21.

19. Tiggemann CL, Dias CP, Radaelli R, Massa JC, Bortoluzzi $\mathrm{R}$, Schoenell MC, et al. Effect of traditional resistance and power training using rated perceived exertion for enhancement of muscle strength, power, and functional performance. AGE. 2016; 38 (42): 2-12. 
20. Coelho BS, Souza LK, Bortoluzzi R, Roncada C, Tiggemann CL, Dias CP. Comparação da força e capacidade funcional entre idosos praticantes de musculação, hidroginástica e não praticantes de exercícios. Rev. bras. geriatr. Gerontol. 2014; 17 (3): 497-504.

21. Ribeiro RF, Oliveira SS, Batiston AP, Santos MLM, Medeiros AA. Avaliação do equilíbrio dinâmico em idosos ativos e sedentários. Fisioter. Bras. 2013; 14(5):38287.

22. Vitorino LM, Paskulin LMG, Vianna L AC. Qualidade de vida de idosos da comunidade e de instituições de longa permanência: estudo comparativo. Rev. Latino-Am. Enfermagem. 2013; 21(SPE): 3-11.

23. Ramos LJ. Avaliação do estado nutricional, de seis domínios da qualidade de vida e da capacidade de tomar decisão de idosos institucionalizados e nãoinstitucionalizados no município de Porto alegre RS. [dissertação]. Porto Alegre: Pontifícia Universidade Católica do Rio Grande do Sul; 2008.

24. Dagios P, Vasconcellos C, Evangelista DHR. Avaliação da qualidade de vida: comparação entre idosos não institucionalizados participantes de um centro de convivência e idosos institucionalizados em JI - Paraná/ RO. Estud. interdiscip. Envelhec. 2015; 20 (2): 469-84.

25. Brunoni L, Schuch FB, Dias CP, Kruel LFM, Tiggemann $\mathrm{CL}$. Treinamento de força diminui os sintomas depressivos e melhora a qualidade de vida relacionada a saúde em idosas. Rev. Bras. Educ. Fís. Esp. 2015; 29 (2): $189-96$.
26. Silva MF, Goulart NBA, Lanferdini FJ, Marcon M, Dias CP. Relação entre os níveis de atividade física e qualidade de vida de idosos sedentários e fisicamente ativos. Rev. bras. geriatr. Gerontol. 2012; $15(4$ ):63442.

27. DipietroL. Physical activity in aging: Changes in patterns and their relationship to health and function. Journals of Gerontology - Series A Biological Sciences and Medical Sciences. 2001; 56(SPEC):13-22.

28. RantanenT, Era P, Heikkinen E. Physical activity and the changes in maximal isometric strength in men and women from the age of 75 to 80 years. Journal of the American Geriatrics Society. 1997; 45(12):1439-45+535.

29. Mazzeo RS, Cavanagh P, Evans WJ, Fiatarone MA, Hagberg J, McAuley E, et al. Exercício e atividade física para pessoas idosas. Rev Bras Ativ Saúde. 1998: $3(1): 48-78$.

\section{Correspondência}

Jéssica Luana Dornelles da Costa

Rua Belém $n^{\circ}: 112$, Centro

Fazenda Vilanova - RS - 95875-000

E-mail: jluana@univates.br 